\title{
Picard iterative processes for initial value problems of singular fractional differential equations
}

Xiaohui Yang ${ }^{1}$ and Yuji Liu ${ }^{2 *}$

${ }^{\text {*Correspondence: }}$

liuyuji888@sohu.com

2Department of Mathematics, Guangdong University of Finance

and Economics, Guangzhou, 510320, P.R. China

Full list of author information is available at the end of the article

\begin{abstract}
In this paper, the initial value problems of singular fractional differential equations are discussed. New criteria on the existence and uniqueness of solutions are obtained. The well-known Picard iterative technique is then extended for fractional differential equations which provides computable sequences that converge uniformly to the solution of the problems discussed. We obtain not only the existence and uniqueness of solutions for the problems, but we also establish iterative schemes for uniformly approximating the solutions. Two examples are given to illustrate the main theorems. MSC: $34 \mathrm{~K} 05 ; 34 \mathrm{~A} 12 ; 34 \mathrm{~A} 40$
\end{abstract}

Keywords: initial value problem; singular fractional differential equation; the Riemann-Liouville fractional derivative; the Caputo fractional derivative; Picard iterative method

\section{Introduction}

Fractional differential equations have been proved to be new and valuable tools in the modeling of many phenomena in various fields of engineering, physics, and economics. We can find numerous applications in viscoelasticity, electrochemistry, control, and electromagnetism. There has been a significant development in fractional differential equations; see the text books $[1,2]$ and the references therein.

Consider the initial value problems (IVP) for the fractional differential equation

$$
\left\{\begin{array} { l } 
{ { } ^ { c } D _ { t _ { 0 } ^ { + } } ^ { \alpha } x = f ( t , x ( t ) ) , \quad t > t _ { 0 } , } \\
{ x ( t _ { 0 } ) = x _ { 0 } , }
\end{array} \text { and } \left\{\begin{array}{l}
D_{t_{0}^{+}}^{\alpha} x=f(t, x(t)), \quad t>t_{0}, \\
I_{t_{0}^{+}}^{1-\alpha} x\left(t_{0}\right)=x_{0},
\end{array}\right.\right.
$$

where $\alpha \in(0,1), x_{0} \in R,{ }^{c} D_{t_{0}^{+}}^{\alpha}$ is the fractional derivative in the sense of Caputo's definition, $D_{t_{0}^{+}}^{\alpha}$ is the fractional derivative in the sense of Riemann-Liouville's definition, $I_{t_{0}^{+}}^{1-\alpha}$ is the fractional integral in the sense of Riemann-Liouville's definition, the function $f$ is defined on $R \times R$. The local existence and uniqueness of solutions of IVP (1) were studied in [3-10].

Let $a>0$ and $b>0$, denote $J=\left[t_{0}-a, t_{0}+a\right], J_{1}=\left[t_{0}, t_{0}+b\right], B=\left[x_{0}-b, x_{0}+b\right]$ and $E=J \times B$. In 2008, Lakshmikantham and Vatsala [5] gave the following existence result for IVP (1).

Theorem A ([5]) Assume that $f \in C\left(J_{1} \times B, R\right)$ and let $|f(t, x)| \leq M$ on $J_{1} \times B$. Then the IVP (1) possesses at least one solution $x(t)$ on $\left[t_{0}, t_{0}+h\right]$, where $h=\min \left\{a,\left(\frac{b}{M} \Gamma(\alpha+1)\right)^{1 / \alpha}\right\}$. 
In 2007, Lin [6] obtained the following local existence results for IVP (1).

Theorem B ([6]) Assume that the function $f: E \rightarrow R$ satisfies the following conditions:

(H1) $f(t, x)$ is Lebesgue measurable with respect to $t$ on $J$;

(H2) $f(t, x)$ is continuous with respect to $x$ on $B$;

(H3) there exists a real-valued function $m \in L^{2}(J)$ such that $|f(t, x)| \leq m(t)$ for almost every $t \in J$ and all $x \in B$.

Then, for $1 / 2<\alpha<1$, there at least exists a solution of the IVP (1) on the interval $\left[t_{0}-h\right.$, $\left.t_{0}+h\right]$ for some positive number $h$.

Theorem C ([6]) All the assumptions of Theorem B hold. Assume that

(H4) there exists a real-valued function $\mu \in L^{4}(J)$ such that $|f(t, x)-f(t, y)| \leq \mu(t)|x-y|$ for almost every $t \in J$ and all $x, y \in B$.

Then, for $1 / 2<\alpha<1$, there exists a unique solution of the IVP (1) on $\left[t_{0}-h, t_{0}+h\right]$ with some positive number $h$.

In Remark 2.3 of [6], it is mentioned that Theorems B and C could be generalized to the case where $\alpha \in(0,1 / 2)$ provided that $m$ is bounded on $\left[t_{0}-a, t_{0}+a\right]$.

In 2009, Zhou [7] proved the following local existence results for IVP (1).

Theorem D ([7]) Assume that the function $f: E \rightarrow R$ satisfies the following conditions of Caratheodory type:

(i) $f(t, x)$ is Lebesgue measurable with respect to $t$ on $J$;

(ii) $f(t, x)$ is continuous with respect to $x$ on $B$;

(iii) there exist a constant $\beta \in(0, \alpha)$ and a real-valued function $m \in L^{1 / \beta}(J)$ such that $|f(t, x)| \leq m(t)$ for almost every $t \in J$ and all $x \in B$.

Then, for $\alpha \in(0,1)$, there at least exists a solution of the IVP (1) on the interval $\left[t_{0}-h\right.$, $\left.t_{0}+h\right]$, where $h=\min \left\{a,\left(\frac{b \Gamma(\alpha)}{M}\left(\frac{\alpha-\beta}{1-\beta}\right)^{1-\beta}\right)^{1 /(\alpha-\beta)}\right\}$ and $M=\left(\int_{t_{0}}^{t_{0}+a}(m(s))^{1 / \beta} d s\right)^{\beta}$.

Theorem E ([7]) All the assumptions of Theorem D hold. Assume that

(iv) there exist a constant $\gamma \in(0, \alpha)$ and a real-valued function $\mu \in L^{1 / \gamma}(J)$ such that $|f(t, x)-f(t, y)| \leq \mu(t)|x-y|$ for almost every $t \in J$ and all $x, y \in B$.

Then, for $\alpha \in(0,1)$, there exists a unique solution of the IVP (1) on the interval $\left[t_{0}-h_{1}\right.$, $\left.t_{0}+h_{1}\right]$, where $h=\min \left\{a,\left(\frac{b \Gamma(\alpha)}{M_{1}}\left(\frac{\alpha-\gamma}{1-\gamma}\right)^{1-\gamma}\right)^{1 /(\alpha-\gamma)}\right\}$ and $M=\left(\int_{t_{0}}^{t_{0}+a}(\mu(s))^{1 / \gamma} d s\right)^{\gamma}$.

One finds that the existence and uniqueness of solution of (1) were proved, but the iterative scheme for uniformly approximating the solutions of (1) was not given in [5-7]. Indeed, often it is very hard to solve fractional differential equations, so we do need a numerical process that can approximate the solution.

Motivated by this reason, in this paper, by using some different methods and new techniques, we obtain criteria on existence and uniqueness of solutions for the following IVPs:

$$
\left\{\begin{array}{l}
{ }^{c} D_{t_{0}^{+}}^{\alpha} x=f(t, x(t)), \quad t>t_{0}, \\
x\left(t_{0}\right)=x_{0}
\end{array}\right.
$$


and

$$
\left\{\begin{array}{l}
D_{t_{0}^{+}}^{\alpha} x=f(t, x(t)), \quad t>t_{0}, \\
\lim _{t \rightarrow t_{0}}\left(t-t_{0}\right)^{1-\alpha} x(t)=x_{0}
\end{array}\right.
$$

where $\alpha \in(0,1], x_{0} \in R$ is a initial value, the fractional derivative ${ }^{c} D_{t_{0}^{+}}^{\alpha}$ is in the sense of Caputo's definition, $D_{t_{0}^{+}}^{\alpha}$ is in the sense of Riemann-Liouville's definition, the function $f$ satisfies assumption given in Section 2 and may be singular at $t=t_{0}$.

A function $x$ is said to be a solution of IVP (2) if there exists $h>0$ such that $x \in C^{0}\left[t_{0}\right.$, $\left.t_{0}+h\right]$ satisfies the equation ${ }^{c} D_{t_{0}^{+}}^{\alpha} x(t)=f(t, x(t))$ a.e. on $\left(t_{0}, t_{0}+h\right]$, and the condition $x\left(t_{0}\right)=x_{0}$. A function $x$ is said to be a solution of IVP (3) if there exists $h>0$ such that $x \in C^{0}\left(t_{0}, t_{0}+h\right]$ satisfies the equation $D_{t_{0}^{+}}^{\alpha} x(t)=f(t, x(t))$ a.e. on $\left(t_{0}, t_{0}+h\right]$, and the condition $\lim _{t \rightarrow t_{0}}\left(t-t_{0}\right)^{1-\alpha} x(t)=x_{0}$.

Our results improve/extend Theorems A, B, C, D and E by generalizing the restrictive condition imposed on $f$. Without the assumption of the existence of lower and upper solution and the monotonic properties of $f(t, x)$, we obtain not only the existence and uniqueness of solutions for the problems, but also we establish iterative schemes for uniformly approximating the solutions.

The remainder of the paper is organized as follows: the main results are given in Section 2, examples are given in Section 3 and a conclusion is given in Section 4.

\section{Main results}

In this section, we prove our main results. Let the beta and gamma functions be defined, respectively, by

$$
\Gamma(x)=\int_{0}^{+\infty} s^{x-1} e^{-s} d s, \quad \mathbf{B}(x, y)=\int_{0}^{1}(1-s)^{x-1} s^{y-1} d s, \quad x>0, y>0 .
$$

Definition 2.1 ([3]) The Riemann-Liouville fractional integral of order $\alpha>0$ of a function $f:(0, \infty) \rightarrow R$ is given by

$$
I_{0+}^{\alpha} f(t)=\frac{1}{\Gamma(\alpha)} \int_{0}^{t}(t-s)^{\alpha-1} f(s) d s
$$

provided that the right-hand side exists.

Definition 2.2 ([3]) The Riemann-Liouville fractional derivative of order $\alpha>0$ of a continuous function $f:(0, \infty) \rightarrow R$ is given by

$$
D_{0^{+}}^{\alpha} f(t)=\frac{1}{\Gamma(n-\alpha)} \frac{d^{n}}{d t^{n}} \int_{0}^{t} \frac{f(s)}{(t-s)^{\alpha-n+1}} d s,
$$

where $n-1<\alpha \leq n$, provided that the right-hand side is point-wise defined on $(0, \infty)$.

Definition 2.3 ([3]) The Caputo fractional derivative of order $\alpha>0$ of a continuous function $f:(0, \infty) \rightarrow R$ is given by

$$
D_{0^{+}}^{\alpha} f(t)=\frac{1}{\Gamma(n-\alpha)} \int_{0}^{t} \frac{f^{(n)}(s)}{(t-s)^{\alpha-n+1}} d s,
$$

where $n-1<\alpha \leq n$, provided that the right-hand side is point-wise defined on $(0, \infty)$. 
For $a>0$ and $b>0$, denote $J=\left[t_{0}, t_{0}+a\right], J_{1}=\left(t_{0}, t_{0}+a\right], B=\left[x_{0}-b, x_{0}+b\right]$ and $B_{t}=$ $\left\{x:\left|x\left(t-t_{0}\right)^{1-\alpha}-x_{0}\right| \leq b\right\}$ for $t \in J_{1}$. The following assumptions will be used in the main results:

(A1) $(t, x) \rightarrow f(t, x)$ is defined on $J_{1} \times B$ and satisfies

(i) $x \rightarrow f(t, x)$ is continuous on $B$ for all $t \in J_{1}, t \rightarrow f(t, x)$ is measurable on $J_{1}$ for all $x \in B$;

(ii) there exist $k>-\alpha$ and $M \geq 0$ such that $|f(t, x)| \leq M\left(t-t_{0}\right)^{k}$ holds for all $t \in J_{1}$ and $x \in B$.

(A2) $(t, x) \rightarrow f\left(t,\left(t-t_{0}\right)^{\alpha-1} x\right)$ is defined on $J_{1} \times B_{t}$ satisfies

(i) $x \rightarrow f\left(t,\left(t-t_{0}\right)^{\alpha-1} x\right)$ is continuous on $B_{t}$ for all $t \in J_{1}, t \rightarrow f\left(t,\left(t-t_{0}\right)^{\alpha-1} x\right)$ is measurable on $J_{1}$ for all $x \in B_{t}$;

(ii) there exist $k>-1$ and $M \geq 0$ such that $\left|f\left(t,\left(t-t_{0}\right)^{\alpha-1} x\right)\right| \leq M\left(t-t_{0}\right)^{k}$ holds for all $t \in J_{1}$ and $x \in B_{t}$.

Choose $h_{1}=\min \left\{a,\left(\frac{b}{M} \frac{\Gamma(\alpha)}{\mathbf{B}(\alpha, k+1)}\right)^{1 /(\alpha+k)}\right\}$. The first result is as follows:

Theorem 2.1 Suppose that (A1) holds and there exist $k>-\alpha$ and $L>0$ such that $\mid f\left(t, x_{1}\right)-$ $f\left(t, x_{2}\right)\left|\leq L\left(t-t_{0}\right)^{k}\right| x_{1}-x_{2} \mid$ for all $t \in\left(t_{0}, t_{0}+h_{1}\right]$ and $x_{1}, x_{2} \in B$. Then IVP (2) has a unique solution $\phi$ defined on $J=\left[t_{0}, t_{0}+h_{1}\right]$ and $\lim _{n \rightarrow \infty} \phi_{n}(t)$ with

$$
\begin{aligned}
& \phi_{0}(t)=x_{0}, \quad t \in\left[t_{0}, t_{0}+h_{1}\right] \\
& \phi_{n}(t)=x_{0}+\int_{t_{0}}^{t} \frac{(t-s)^{\alpha-1}}{\Gamma(\alpha)} f\left(s, \phi_{n-1}(s)\right) d s, \quad t \in\left[t_{0}, t_{0}+h_{1}\right], n=1,2, \ldots
\end{aligned}
$$

Choose $h_{2}=\min \left\{a,\left(\frac{b}{M} \frac{\Gamma(\alpha)}{\mathbf{B}(\alpha, k+1)}\right)^{1 /(1+k)}\right\}$. The second result is as follows.

Theorem 2.2 Suppose that (A2) holds and there exist $k>-1$ and $L>0$ such that $\mid f(t$, $\left.\left(t-t_{0}\right)^{\alpha-1} x_{1}\right)-f\left(t,\left(t-t_{0}\right)^{\alpha-1} x_{2}\right)\left|\leq L\left(t-t_{0}\right)^{k}\right| x_{1}-x_{2} \mid$ for all $t \in\left(t_{0}, t_{0}+h_{2}\right]$ and $x_{1}, x_{2} \in B_{1}$. Then IVP (3) has a unique solution $\phi$ defined on $\left(t_{0}, t_{0}+h_{2}\right]$ and $\phi(t)=\left(t-t_{0}\right)^{\alpha-1} \lim _{n \rightarrow \infty}(t-$ $\left.t_{0}\right)^{1-\alpha} \phi_{n}(t)$ with

$$
\begin{aligned}
& \phi_{0}(t)=x_{0}\left(t-t_{0}\right)^{\alpha-1}, \quad t \in\left(t_{0}, t_{0}+h_{2}\right], \\
& \phi_{n}(t)=x_{0}\left(t-t_{0}\right)^{\alpha-1}+\int_{t_{0}}^{t} \frac{(t-s)^{\alpha-1}}{\Gamma(\alpha)} f\left(s, \phi_{n-1}(s)\right) d s, \quad t \in\left(t_{0}, t_{0}+h_{2}\right], n=1,2, \ldots
\end{aligned}
$$

Lemma 2.1 Suppose that (A1) holds. Then $x:\left[t_{0}, t_{0}+h_{1}\right] \rightarrow R$ is a solution of IVP (2) if and only if $x:\left[t_{0}, t_{0}+h_{1}\right] \rightarrow R$ is a solution of the following integral equation:

$$
x(t)=x_{0}+\int_{t_{0}}^{t} \frac{(t-s)^{\alpha-1}}{\Gamma(\alpha)} f(s, x(s)) d s .
$$

Proof Suppose that $x:\left[t_{0}, t_{0}+h_{1}\right] \rightarrow R$ is a solution of IVP (2). Since $f$ is defined in $J_{1} \times B$, then $\left|x(t)-x_{0}\right| \leq b$ for all $t \in\left[t_{0}, t_{0}+h_{1}\right]$. From (A1), there exist $k>-\alpha$ and $M \geq 0$ such that $|f(t, x(t))| \leq M\left(t-t_{0}\right)^{k}$ for all $t \in\left(t_{0}, t_{0}+h_{1}\right]$. Then we have

$$
\left|\int_{t_{0}}^{t} \frac{(t-s)^{\alpha-1}}{\Gamma(\alpha)} f(s, x(s)) d s\right| \leq \int_{t_{0}}^{t} \frac{(t-s)^{\alpha-1}}{\Gamma(\alpha)} M_{r}\left(s-t_{0}\right)^{k} d s=M_{r}\left(t-t_{0}\right)^{\alpha+k} \frac{\mathbf{B}(\alpha, k+1)}{\Gamma(\alpha)}
$$


It is easy to see that

$$
x(t)=x_{0}+\int_{t_{0}}^{t} \frac{(t-s)^{\alpha-1}}{\Gamma(\alpha)} f(s, x(s)) d s, \quad t \in\left(t_{0}, t_{0}+h_{1}\right] .
$$

Since $k>-\alpha$, then $x \in C^{0}\left[t_{0}, t_{0}+h_{1}\right]$ is a solution of (4). On the other hand, it is easy to see that $x:\left[t_{0}, t_{0}+h_{1}\right] \rightarrow R$ is a solution of (4) implies that $x$ is a solution of IVP (2) defined on $\left[t_{0}, t_{0}+h_{1}\right]$. The proof is completed.

Choose a Picard function sequence as

$$
\begin{aligned}
& \phi_{0}(t)=x_{0}, \quad t \in\left[t_{0}, t_{0}+h_{1}\right] \\
& \phi_{n}(t)=x_{0}+\int_{t_{0}}^{t} \frac{(t-s)^{\alpha-1}}{\Gamma(\alpha)} f\left(s, \phi_{n-1}(s)\right) d s, \quad t \in\left[t_{0}, t_{0}+h_{1}\right], n=1,2, \ldots
\end{aligned}
$$

Lemma 2.2 Suppose that (A1) holds. Then $\phi_{n}$ is continuous on $\left[t_{0}, t_{0}+h_{1}\right]$ and satisfies $\left|\phi_{n}(t)-x_{0}\right| \leq b$.

Proof From (A1), there exist $k>-\alpha$ and $M \geq 0$ such that $|f(t, x)| \leq M\left(t-t_{0}\right)^{k}$ for all $t \in J_{1}$ and $\left|x-x_{0}\right| \leq b$. If $n=1$, then $\phi_{1}(t)=x_{0}+\int_{t_{0}}^{t} \frac{(t-s)^{\alpha-1}}{\Gamma(\alpha)} f\left(s, x_{0}\right) d s$. Then

$$
\left|\int_{t_{0}}^{t} \frac{(t-s)^{\alpha-1}}{\Gamma(\alpha)} f\left(s, x_{0}\right) d s\right| \leq \int_{t_{0}}^{t} \frac{(t-s)^{\alpha-1}}{\Gamma(\alpha)} M\left(s-t_{0}\right)^{k} d s=M\left(t-t_{0}\right)^{\alpha+k} \frac{\mathbf{B}(\alpha, k+1)}{\Gamma(\alpha)}
$$

It is easy to see that $\phi_{1} \in C^{0}\left[t_{0}, t_{0}+h_{1}\right]$ and

$$
\left|\phi_{1}(t)-x_{0}\right| \leq M\left(t-t_{0}\right)^{\alpha+k} \frac{\mathbf{B}(\alpha, k+1)}{\Gamma(\alpha)} \leq M h_{1}^{\alpha+k} \frac{\mathbf{B}(\alpha, k+1)}{\Gamma(\alpha)}=b, \quad t \in\left[t_{0}, t_{0}+h_{1}\right] .
$$

Now suppose that $\phi_{n} \in C^{0}\left[t_{0}, t_{0}+h_{1}\right]$ and $\left|\phi_{n}(t)-x_{0}\right| \leq b$ for all $t \in\left[t_{0}, t_{0}+h_{1}\right]$. By

$$
\phi_{n+1}(t)=x_{0}+\int_{t_{0}}^{t} \frac{(t-s)^{\alpha-1}}{\Gamma(\alpha)} f\left(s, \phi_{n}(s)\right) d s
$$

It is easy to see that $\phi_{n+1} \in C^{0}\left[t_{0}, t_{0}+h_{1}\right]$ and

$$
\begin{aligned}
\left|\phi_{n+1}(t)-x_{0}\right| & \leq \int_{t_{0}}^{t} \frac{(t-s)^{\alpha-1}}{\Gamma(\alpha)} M\left(s-t_{0}\right)^{k} d s=M\left(t-t_{0}\right)^{\alpha+k} \frac{\mathbf{B}(\alpha, k+1)}{\Gamma(\alpha)} \\
& \leq M h_{1}^{\alpha+k} \frac{\mathbf{B}(\alpha, k+1)}{\Gamma(\alpha)} \leq b .
\end{aligned}
$$

So the result is correct when $n+1$. Then by the mathematical induction method, the result holds for all $n$.

Lemma 2.3 Suppose that $x>0$. Then $\Gamma(x)=\lim _{m \rightarrow+\infty} \frac{m^{x} m !}{x(x+1)(x+2) \cdots(x+m)}$.

Proof The proof can be found in [2] and is omitted. 
Lemma 2.4 Suppose that (A1) holds and there exists $L>0$ such that $\left|f\left(t, x_{1}\right)-f\left(t, x_{2}\right)\right| \leq$ $L\left(t-t_{0}\right)^{k}\left|x_{1}-x_{2}\right|$ for all $t \in\left(t_{0}, t_{0}+h_{1}\right]$ and $x_{1}, x_{2} \in B$. Then $\left\{\phi_{n}(t)\right\}$ is convergent uniformly on $\left[t_{0}, t_{0}+h_{1}\right]$.

Proof Consider

$$
\phi_{0}(t)+\left[\phi_{1}(t)-\phi_{0}(t)\right]+\left[\phi_{2}(t)-\phi_{1}(t)\right]+\cdots+\left[\phi_{n}(t)-\phi_{n-1}(t)\right]+\cdots, \quad t \in\left[t_{0}, t_{0}+h_{1}\right] .
$$

We have by the proof of Lemma 2.2

$$
\left|\phi_{1}(t)-\phi_{0}(t)\right| \leq M\left(t-t_{0}\right)^{\alpha+k} \frac{\mathbf{B}(\alpha, k+1)}{\Gamma(\alpha)}, \quad t \in\left[t_{0}, t_{0}+h_{1}\right] .
$$

Now, we have by Lemma 2.2

$$
\begin{aligned}
\left|\phi_{2}(t)-\phi_{1}(t)\right| & \leq \int_{t_{0}}^{t} \frac{(t-s)^{\alpha-1}}{\Gamma(\alpha)}\left|f\left(s, \phi_{1}(s)\right)-f\left(s, \phi_{0}(s)\right)\right| d s \\
& \leq \int_{t_{0}}^{t} \frac{(t-s)^{\alpha-1}}{\Gamma(\alpha)} L\left(s-t_{0}\right)^{k}\left|\phi_{1}(s)-\phi_{0}(s)\right| d s \\
& \leq \int_{t_{0}}^{t} \frac{(t-s)^{\alpha-1}}{\Gamma(\alpha)} L\left(s-t_{0}\right)^{k} M\left(s-t_{0}\right)^{\alpha+k} \frac{\mathbf{B}(\alpha, k+1)}{\Gamma(\alpha)} d s \\
& =L M \frac{\mathbf{B}(\alpha, k+1)}{\Gamma(\alpha)} \int_{t_{0}}^{t} \frac{(t-s)^{\alpha-1}}{\Gamma(\alpha)}\left(s-t_{0}\right)^{\alpha+2 k} d s \\
& =L M \frac{\mathbf{B}(\alpha, k+1)}{\Gamma(\alpha)} \frac{\mathbf{B}(\alpha, \alpha+2 k+1)}{\Gamma(\alpha)}\left(t-t_{0}\right)^{2 \alpha+2 k} .
\end{aligned}
$$

Now suppose that

$$
\left|\phi_{n+1}(t)-\phi_{n}(t)\right| \leq L^{n} M\left(t-t_{0}\right)^{(n+1) \alpha+(n+1) k} \prod_{i=1}^{n} \frac{\mathbf{B}(\alpha, i \alpha+(i+1) k+1)}{\Gamma(\alpha)} .
$$

We have

$$
\begin{aligned}
& \left|\phi_{n+2}(t)-\phi_{n+1}(t)\right| \\
& \quad \leq \int_{t_{0}}^{t} \frac{(t-s)^{\alpha-1}}{\Gamma(\alpha)}\left|f\left(s, \phi_{n+1}(s)\right)-f\left(s, \phi_{n}(s)\right)\right| d s \\
& \quad \leq \int_{t_{0}}^{t} \frac{(t-s)^{\alpha-1}}{\Gamma(\alpha)} L\left(s-t_{0}\right)^{k}\left|\phi_{n+1}(s)-\phi_{n}(s)\right| d s \\
& \quad \leq \int_{t_{0}}^{t} \frac{(t-s)^{\alpha-1}}{\Gamma(\alpha)} L\left(s-t_{0}\right)^{k} L^{n} M\left(t-t_{0}\right)^{(n+1) \alpha+(n+1) k} \prod_{i=1}^{n} \frac{\mathbf{B}(\alpha, i \alpha+(i+1) k+1)}{\Gamma(\alpha)} d s \\
& \quad=L^{n+1} M\left(t-t_{0}\right)^{(n+2) \alpha+(n+2) k} \prod_{i=1}^{n+1} \frac{\mathbf{B}(\alpha, i \alpha+(i+1) k+1)}{\Gamma(\alpha)} .
\end{aligned}
$$


So the result is correct when $n+1$. Then by the mathematical induction method, we get

$$
\begin{aligned}
\left|\phi_{n+2}(t)-\phi_{n+1}(t)\right| & \leq L^{n+1} M\left(t-t_{0}\right)^{(n+2) \alpha+(n+2) k} \prod_{i=1}^{n+1} \frac{\mathbf{B}(\alpha, i \alpha+(i+1) k+1)}{\Gamma(\alpha)} \\
& \leq L^{n+1} M h_{1}^{(n+2) \alpha+(n+2) k} \prod_{i=1}^{n+1} \frac{\mathbf{B}(\alpha, i \alpha+(i+1) k+1)}{\Gamma(\alpha)} \\
& =M L^{n+1} h_{1}^{(n+2)(\alpha+k)} \prod_{i=1}^{n+1} \frac{\Gamma(i \alpha+(i+1) k+1)}{\Gamma((i+1) \alpha+(i+1) k+1)} .
\end{aligned}
$$

Consider

$$
\sum_{n=1}^{\infty} u_{n}=: \sum_{n=1}^{\infty} M L^{n+1} h_{1}^{(n+2)(\alpha+k)} \prod_{i=1}^{n+1} \frac{\Gamma(i \alpha+(i+1) k+1)}{\Gamma((i+1) \alpha+(i+1) k+1)} .
$$

By Lemma 2.3, we get

$$
\begin{aligned}
\frac{u_{n+1}}{u_{n}}= & L h_{1}^{\alpha+k} \frac{\Gamma((n+2) \alpha+(n+3) k+1)}{\Gamma((n+3) \alpha+(n+3) k+1)} \\
= & L h_{1}^{\alpha+k} \frac{\lim _{m \rightarrow+\infty} \frac{m^{(n+2) \alpha+(n+3) k+1} m !}{((n+2) \alpha+(n+3) k+1)((n+2) \alpha+(n+3) k+2) \cdots((n+2) \alpha+(n+3) k+1+m)}}{\lim _{m \rightarrow+\infty} \frac{m^{(n+3) \alpha+(n+3) k+1} m !}{((n+3) \alpha+(n+3) k+1)((n+3) \alpha+(n+3) k+2) \cdots((n+3) \alpha+(n+3) k+1+m)}} \\
= & L h_{1}^{\alpha+k} \lim _{m \rightarrow+\infty} \frac{\frac{m^{(n+2) \alpha+(n+3) k+1} m !}{((n+2) \alpha+(n+3) k+1)((n+2) \alpha+(n+3) k+2) \cdots((n+2) \alpha+(n+3) k+1+m)}}{\frac{m^{(n+3) \alpha+(n+3) k+1} m !}{(n+3) \alpha+(n+3) k+1)((n+3) \alpha+(n+3) k+2) \cdots((n+3) \alpha+(n+3) k+1+m)}} \\
= & L h_{1}^{\alpha+k} \lim _{m \rightarrow+\infty} m^{-\alpha}(((n+2) \alpha+(n+3) k+1)((n+2) \alpha+(n+3) k+2) \cdots \\
& \times((n+2) \alpha+(n+3) k+1+m)) \\
& /(((n+3) \alpha+(n+3) k+1)((n+3) \alpha+(n+3) k+2) \cdots \\
& \times((n+3) \alpha+(n+3) k+1+m)) .
\end{aligned}
$$

One sees that

$$
\frac{((n+2) \alpha+(n+3) k+1)((n+2) \alpha+(n+3) k+2) \cdots((n+2) \alpha+(n+3) k+1+m)}{((n+3) \alpha+(n+3) k+1)((n+3) \alpha+(n+3) k+2) \cdots((n+3) \alpha+(n+3) k+1+m)}
$$

is bounded for all $m, n$. Then $\lim _{n \rightarrow \infty} \frac{u_{n+1}}{u_{n}}=0$. Then $\sum_{n=1}^{\infty} u_{n}$ is convergent. Hence

$$
\phi_{0}(t)+\left[\phi_{1}(t)-\phi_{0}(t)\right]+\left[\phi_{2}(t)-\phi_{1}(t)\right]+\cdots+\left[\phi_{n}(t)-\phi_{n-1}(t)\right]+\cdots, \quad t \in\left[t_{0}, t_{0}+h_{1}\right]
$$

is uniformly convergent. Then $\left\{\phi_{n}(t)\right\}$ is convergent uniformly on $\left[t_{0}, t_{0}+h_{1}\right]$. The proof is complete.

Lemma 2.5 Suppose that (A1) holds and there exists $L>0$ such that $\left|f\left(t, x_{1}\right)-f\left(t, x_{2}\right)\right| \leq$ $L\left(t-t_{0}\right)^{k}\left|x_{1}-x_{2}\right|$ for all $t \in\left(t_{0}, t_{0}+h_{1}\right]$ and $x_{1}, x_{2} \in B$. Then $\phi(t)=\lim _{n \rightarrow \infty} \phi_{n}(t)$ is a unique continuous solution of $(4)$ defined on $\left[t_{0}, t_{0}+h_{1}\right]$. 
Proof By $\phi(t)=\lim _{n \rightarrow \infty} \phi_{n}(t)$ and Lemma 2.2, we see $\left|\phi(t)-x_{0}\right| \leq b$. Then

$$
\left|f\left(t, \phi_{n}(t)\right)-f(t, \phi(t))\right| \leq L\left(t-t_{0}\right)^{k}\left|\phi_{n}(t)-\phi(t)\right|, \quad t \in\left(t_{0}, t_{0}+h_{1}\right] .
$$

It follows that

$$
\begin{aligned}
& \left(t-t_{0}\right)^{-k}\left|f\left(t, \phi_{n}(t)\right)-f(t, \phi(t))\right| \leq L\left|\phi_{n}(t)-\phi(t)\right| \rightarrow 0 \\
& \quad \text { uniformly as } n \rightarrow \infty \text { on }\left(t_{0}, t_{0}+h_{1}\right] .
\end{aligned}
$$

Hence

$$
\begin{aligned}
\phi(t) & =\lim _{n \rightarrow \infty} \phi_{n}(t)=\lim _{n \rightarrow \infty}\left[x_{0}+\int_{t_{0}}^{t} \frac{(t-s)^{\alpha-1}}{\Gamma(\alpha)} f\left(s, \phi_{n-1}(s)\right) d s\right] \\
& =x_{0}+\lim _{n \rightarrow \infty} \int_{t_{0}}^{t} \frac{(t-s)^{\alpha-1}}{\Gamma(\alpha)} f\left(s, \phi_{n-1}(s)\right) d s \\
& =x_{0}+\lim _{n \rightarrow \infty} \int_{t_{0}}^{t} \frac{(t-s)^{\alpha-1}}{\Gamma(\alpha)}\left(s-t_{0}\right)^{k}\left(s-t_{0}\right)^{-k} f\left(s, \phi_{n-1}(s)\right) d s \\
& =x_{0}+\int_{t_{0}}^{t} \frac{(t-s)^{\alpha-1}}{\Gamma(\alpha)}\left(s-t_{0}\right)^{k} \lim _{n \rightarrow \infty}\left(s-t_{0}\right)^{-k} f(s, \phi(s)) d s \\
& =x_{0}+\int_{t_{0}}^{t} \frac{(t-s)^{\alpha-1}}{\Gamma(\alpha)} f(s, \phi(s)) d s .
\end{aligned}
$$

Then $\phi$ is a unique continuous solution of (4) defined on $\left[t_{0}, t_{0}+h_{1}\right]$.

Suppose that $\psi$ defined on $\left[t_{0}, t_{0}+h_{1}\right]$ is also a solution of (4). Then $\left|\psi(t)-x_{0}\right| \leq b$ for all $t \in\left[t_{0}, t_{0}+h_{1}\right]$ and

$$
\psi(t)=x_{0}+\int_{t_{0}}^{t} \frac{(t-s)^{\alpha-1}}{\Gamma(\alpha)} f(s, \phi(s)) d s, \quad t \in\left[t_{0}, t_{0}+h_{1}\right]
$$

We need to prove that $\phi(t) \equiv \psi(t)$ on $\left[t_{0}, t_{0}+h_{1}\right]$. By (A1), there exist $k>-\alpha$ and $M \geq 0$ such that $|f(t, \psi(t))| \leq M\left(t-t_{0}\right)^{k}$ for all $t \in\left(t_{0}, t_{0}+h_{1}\right]$. Then

$$
\begin{aligned}
\left|\phi_{0}(t)-\psi(t)\right| & =\left|\int_{t_{0}}^{t} \frac{(t-s)^{\alpha-1}}{\Gamma(\alpha)} f(s, \psi(s)) d s\right| \\
& \leq \int_{t_{0}}^{t} \frac{(t-s)^{\alpha-1}}{\Gamma(\alpha)} M\left(s-t_{0}\right)^{k} d s=M\left(t-t_{0}\right)^{\alpha+k} \frac{\mathbf{B}(\alpha, k+1)}{\Gamma(\alpha)} .
\end{aligned}
$$

Furthermore, we have

$$
\begin{aligned}
\left|\phi_{1}(t)-\psi(t)\right| & =\left|\int_{t_{0}}^{t} \frac{(t-s)^{\alpha-1}}{\Gamma(\alpha)}\left(f\left(s, \phi_{0}(s)\right)-f(s, \psi(s))\right) d s\right| \\
& \leq \int_{t_{0}}^{t} \frac{(t-s)^{\alpha-1}}{\Gamma(\alpha)} L\left(s-t_{0}\right)^{k}\left|\phi_{0}(s)-\psi(s)\right| d s \\
& \leq \int_{t_{0}}^{t} \frac{(t-s)^{\alpha-1}}{\Gamma(\alpha)} L\left(s-t_{0}\right)^{k} M\left(s-t_{0}\right)^{\alpha+k} \frac{\mathbf{B}(\alpha, k+1)}{\Gamma(\alpha)} d s \\
& =L M \frac{\mathbf{B}(\alpha, k+1)}{\Gamma(\alpha)} \frac{\mathbf{B}(\alpha, \alpha+2 k+1)}{\Gamma(\alpha)}\left(t-t_{0}\right)^{2 \alpha+2 k} .
\end{aligned}
$$


Now suppose that

$$
\left|\phi_{n}(t)-\psi(t)\right| \leq L^{n} M\left(t-t_{0}\right)^{(n+1) \alpha+(n+1) k} \prod_{i=1}^{n} \frac{\mathbf{B}(\alpha, i \alpha+(i+1) k+1)}{\Gamma(\alpha)} .
$$

Then

$$
\begin{aligned}
& \left|\phi_{n+1}(t)-\psi(t)\right| \\
& \quad=\left|\int_{t_{0}}^{t} \frac{(t-s)^{\alpha-1}}{\Gamma(\alpha)}\left(f\left(s, \phi_{n}(s)\right)-f(s, \psi(s))\right) d s\right| \\
& \quad \leq \int_{t_{0}}^{t} \frac{(t-s)^{\alpha-1}}{\Gamma(\alpha)} L\left(s-t_{0}\right)^{k}\left|\phi_{n}(s)-\psi(s)\right| d s \\
& \quad \leq \int_{t_{0}}^{t} \frac{(t-s)^{\alpha-1}}{\Gamma(\alpha)} L\left(s-t_{0}\right)^{k} L^{n} M\left(s-t_{0}\right)^{(n+1) \alpha+(n+1) k} \prod_{i=1}^{n} \frac{\mathbf{B}(\alpha, i \alpha+(i+1) k+1)}{\Gamma(\alpha)} d s \\
& \quad=L^{n+1} M\left(t-t_{0}\right)^{(n+2) \alpha+(n+2) k} \prod_{i=1}^{n+1} \frac{\mathbf{B}(\alpha, i \alpha+(i+1) k+1)}{\Gamma(\alpha)} .
\end{aligned}
$$

Hence

$$
\begin{aligned}
\left|\phi_{n+1}(t)-\psi(t)\right| & \leq L^{n} M\left(t-t_{0}\right)^{(n+1) \alpha+(n+1) k} \prod_{i=1}^{n} \frac{\mathbf{B}(\alpha, i \alpha+(i+1) k+1)}{\Gamma(\alpha)} \\
& \leq L^{n} M h_{1}^{(n+1) \alpha+(n+1) k} \prod_{i=1}^{n} \frac{\mathbf{B}(\alpha, i \alpha+(i+1) k+1)}{\Gamma(\alpha)} \\
& =L^{n} M h_{1}^{(n+1) \alpha+(n+1) k} \prod_{i=1}^{n} \frac{\Gamma(i \alpha+(i+1) k+1)}{\Gamma((i+1) \alpha+(i+1) k+1)} .
\end{aligned}
$$

From Lemma 2.3 and the proof of Lemma 2.4, we have

$$
\sum_{n=1}^{\infty} L^{n} M h_{1}^{(n+1) \alpha+(n+1) k} \prod_{i=1}^{n} \frac{\Gamma(i \alpha+(i+1) k+1)}{\Gamma((i+1) \alpha+(i+1) k+1)}
$$

is convergent. Then

$$
L^{n} M h_{1}^{(n+1) \alpha+(n+1) k} \prod_{i=1}^{n} \frac{\Gamma(i \alpha+(i+1) k+1)}{\Gamma((i+1) \alpha+(i+1) k+1)} \rightarrow 0 \quad \text { as } n \rightarrow \infty .
$$

Then $\lim _{n \rightarrow \infty} \phi_{n}(t)=\psi(t)$ uniformly on $\left[t_{0}, t_{0}+h_{1}\right]$. Then $\phi(t) \equiv \psi(t)$. The proof is complete.

Proof of Theorem 2.1 From Lemma 2.5 and Lemma 2.1, $\phi(t)=\lim _{n \rightarrow \infty} \phi_{n}(t)$ is a unique continuous solution of IVP (2) defined on $\left[t_{0}, t_{0}+h_{1}\right]$. The proof is ended. 
Lemma 2.6 Suppose that (A2) holds. Then $x:\left[t_{0}, t_{0}+h_{2}\right] \rightarrow R$ is a solution of IVP (3) if and only if $x:\left(t_{0}, t_{0}+h_{2}\right] \rightarrow R$ is a solution of the following integral equation:

$$
x(t)=x_{0}\left(t-t_{0}\right)^{\alpha-1}+\int_{t_{0}}^{t} \frac{(t-s)^{\alpha-1}}{\Gamma(\alpha)} f(s, x(s)) d s .
$$

Proof Suppose that $x:\left(t_{0}, t_{0}+h_{2}\right] \rightarrow R$ is a solution of IVP (3). Then $\left|\left(t-t_{0}\right)^{1-\alpha}\right| x(t)-x_{0} \mid \leq$ $b$ for all $t \in\left(t_{0}, t_{0}+h_{2}\right]$. From (A2), there exist $k>-1$ and $M \geq 0$ such that

$$
|f(t, x(t))|=\left|f\left(t,\left(t-t_{0}\right)^{\alpha-1}\left(t-t_{0}\right)^{1-\alpha} x(t)\right)\right| \leq M\left(t-t_{0}\right)^{k} \quad \text { for all } t \in\left(t_{0}, t_{0}+h_{2}\right] .
$$

Then we have

$$
\left|\int_{t_{0}}^{t} \frac{(t-s)^{\alpha-1}}{\Gamma(\alpha)} f(s, x(s)) d s\right| \leq \int_{t_{0}}^{t} \frac{(t-s)^{\alpha-1}}{\Gamma(\alpha)} M\left(s-t_{0}\right)^{k} d s=M\left(t-t_{0}\right)^{\alpha+k} \frac{\mathbf{B}(\alpha, k+1)}{\Gamma(\alpha)}
$$

Then

$$
\lim _{t \rightarrow t_{0}}\left(t-t_{0}\right)^{1-\alpha} \int_{t_{0}}^{t} \frac{(t-s)^{\alpha-1}}{\Gamma(\alpha)} f(s, x(s)) d s=0
$$

It is easy to see that

$$
x(t)=x_{0}\left(t-t_{0}\right)^{\alpha-1}+\int_{t_{0}}^{t} \frac{(t-s)^{\alpha-1}}{\Gamma(\alpha)} f(s, x(s)) d s, \quad t \in\left(t_{0}, t_{0}+h_{2}\right]
$$

Since $k>-1$, then $x \in C^{0}\left(t_{0}, t_{0}+h_{1}\right]$ is a solution of (6). On the other hand, it is easy to see that $x:\left[t_{0}, t_{0}+h_{2}\right] \rightarrow R$ is a solution of (6) implies that $x$ is a solution of IVP (3) defined on $\left[t_{0}, t_{0}+h_{2}\right]$. The proof is completed.

Choose Picard function sequence as

$$
\begin{aligned}
& \phi_{0}(t)=x_{0}\left(t-t_{0}\right)^{\alpha-1}, \quad t \in\left(t_{0}, t_{0}+h_{2}\right] \\
& \phi_{n}(t)=x_{0}\left(t-t_{0}\right)^{\alpha-1}+\int_{t_{0}}^{t} \frac{(t-s)^{\alpha-1}}{\Gamma(\alpha)} f\left(s, \phi_{n-1}(s)\right) d s, \quad t \in\left(t_{0}, t_{0}+h_{2}\right], n=1,2, \ldots
\end{aligned}
$$

Lemma 2.7 Suppose that (A2) holds. Then $\phi_{n}$ is continuous on $\left(t_{0}, t_{0}+h_{2}\right]$ and satisfies $\left|\left(t-t_{0}\right)^{1-\alpha} \phi_{n}(t)-x_{0}\right| \leq b$.

Proof From (A2), there exist $k>-1$ and $M \geq 0$ such that $\left|f\left(t, t^{\alpha-1} x\right)\right| \leq M\left(t-t_{0}\right)^{k}$ for all $t \in J_{1}$ and $\left|x\left(t-t_{0}\right)^{1-\alpha}-x_{0}\right| \leq b$. If $n=1$, then $\phi_{1}(t)=x_{0}\left(t-t_{0}\right)^{\alpha-1}+\int_{t_{0}}^{t} \frac{(t-s)^{\alpha-1}}{\Gamma(\alpha)} f\left(s, x_{0} s^{\alpha-1}\right) d s$. Then

$$
\left|\int_{t_{0}}^{t} \frac{(t-s)^{\alpha-1}}{\Gamma(\alpha)} f\left(s, \phi_{0}(s)\right) d s\right| \leq \int_{t_{0}}^{t} \frac{(t-s)^{\alpha-1}}{\Gamma(\alpha)} M\left(s-t_{0}\right)^{k} d s=M\left(t-t_{0}\right)^{\alpha+k} \frac{\mathbf{B}(\alpha, k+1)}{\Gamma(\alpha)}
$$


It is easy to see that $\phi_{1} \in C^{0}\left(t_{0}, t_{0}+h_{1}\right]$ and

$$
\begin{aligned}
\left|\left(t-t_{0}\right)^{1-\alpha} \phi_{1}(t)-x_{0}\right| & \leq\left(t-t_{0}\right)^{1-\alpha} M\left(t-t_{0}\right)^{\alpha+k} \frac{\mathbf{B}(\alpha, k+1)}{\Gamma(\alpha)} \\
& \leq M h_{1}^{1+k} \frac{\mathbf{B}(\alpha, k+1)}{\Gamma(\alpha)}=b, \quad t \in\left[t_{0}, t_{0}+h_{2}\right] .
\end{aligned}
$$

Now suppose that $\phi_{n} \in C^{0}\left[t_{0}, t_{0}+h_{2}\right]$ and $\left|\left(t-t_{0}\right)^{1-\alpha} \phi_{n}(t)-x_{0}\right| \leq b$ for all $t \in\left[t_{0}, t_{0}+h_{2}\right]$. By

$$
\phi_{n+1}(t)=x_{0}\left(t-t_{0}\right)^{\alpha-1}+\int_{t_{0}}^{t} \frac{(t-s)^{\alpha-1}}{\Gamma(\alpha)} f\left(s, \phi_{n}(s)\right) d s .
$$

It is easy to see that $\phi_{n+1} \in C^{0}\left(t_{0}, t_{0}+h_{1}\right]$ and

$$
\begin{aligned}
\left|\left(t-t_{0}\right)^{1-\alpha} \phi_{n+1}(t)-x_{0}\right| & \leq\left(t-t_{0}\right)^{1-\alpha} \int_{t_{0}}^{t} \frac{(t-s)^{\alpha-1}}{\Gamma(\alpha)} M\left(s-t_{0}\right)^{k} d s \\
& =M\left(t-t_{0}\right)^{1+k} \frac{\mathbf{B}(\alpha, k+1)}{\Gamma(\alpha)} \leq M h_{1}^{1+k} \frac{\mathbf{B}(\alpha, k+1)}{\Gamma(\alpha)} \leq b .
\end{aligned}
$$

So the result is correct when $n+1$. Then by the mathematical induction method, the result holds for all $n$.

Lemma 2.8 Suppose that (A2) holds and there exists $L>0$ such that $f\left(t,\left(t-t_{0}\right)^{\alpha-1} x_{1}\right)-$ $f\left(t,\left(t-t_{0}\right)^{\alpha-1} x_{2}\right)\left|\leq L\left(t-t_{0}\right)^{k}\right| x_{1}-x_{2} \mid$ for all $t \in\left(t_{0}, t_{0}+h_{2}\right]$ and $x_{1}, x_{2} \in B_{t}$. Then $\{(t-$ $\left.\left.t_{0}\right)^{1-\alpha} \phi_{n}(t)\right\}$ is convergent uniformly on $\left[t_{0}, t_{0}+h_{2}\right]$.

Proof Consider

$$
\begin{aligned}
& \left(t-t_{0}\right)^{1-\alpha} \phi_{0}(t)+\left(t-t_{0}\right)^{1-\alpha}\left[\phi_{1}(t)-\phi_{0}(t)\right]+\cdots+\left(t-t_{0}\right)^{1-\alpha}\left[\phi_{n}(t)-\phi_{n-1}(t)\right]+\cdots, \\
& \quad t \in\left[t_{0}, t_{0}+h_{2}\right] .
\end{aligned}
$$

We have by the proof of Lemma 2.2

$$
\left(t-t_{0}\right)^{1-\alpha}\left|\phi_{1}(t)-\phi_{0}(t)\right| \leq M\left(t-t_{0}\right)^{1+k} \frac{\mathbf{B}(\alpha, k+1)}{\Gamma(\alpha)}, \quad t \in\left[t_{0}, t_{0}+h_{2}\right] .
$$

Now, we have by Lemma 2.2 that

$$
\begin{aligned}
\left(t-t_{0}\right)^{1-\alpha}\left|\phi_{2}(t)-\phi_{1}(t)\right| & \leq\left(t-t_{0}\right)^{1-\alpha} \int_{t_{0}}^{t} \frac{(t-s)^{\alpha-1}}{\Gamma(\alpha)}\left|f\left(s, \phi_{1}(s)\right)-f\left(s, \phi_{0}(s)\right)\right| d s \\
& \leq\left(t-t_{0}\right)^{1-\alpha} \int_{t_{0}}^{t} \frac{(t-s)^{\alpha-1}}{\Gamma(\alpha)} L\left(s-t_{0}\right)^{k}\left|\phi_{1}(s)-\phi_{0}(s)\right| d s \\
& \leq\left(t-t_{0}\right)^{1-\alpha} \int_{t_{0}}^{t} \frac{(t-s)^{\alpha-1}}{\Gamma(\alpha)} L\left(s-t_{0}\right)^{k} M\left(s-t_{0}\right)^{1+k} \frac{\mathbf{B}(\alpha, k+1)}{\Gamma(\alpha)} d s \\
& =L M \frac{\mathbf{B}(\alpha, k+1)}{\Gamma(\alpha)}\left(t-t_{0}\right)^{1-\alpha} \int_{t_{0}}^{t} \frac{(t-s)^{\alpha-1}}{\Gamma(\alpha)}\left(s-t_{0}\right)^{1+2 k} d s \\
& =L M \frac{\mathbf{B}(\alpha, k+1)}{\Gamma(\alpha)} \frac{\mathbf{B}(\alpha, 2 k+2)}{\Gamma(\alpha)}\left(t-t_{0}\right)^{2+2 k} .
\end{aligned}
$$


Now suppose that

$$
\left(t-t_{0}\right)^{1-\alpha}\left|\phi_{n+1}(t)-\phi_{n}(t)\right| \leq L^{n} M\left(t-t_{0}\right)^{(n+1)+(n+1) k} \prod_{i=1}^{n} \frac{\mathbf{B}(\alpha,(i+1)(k+1))}{\Gamma(\alpha)}
$$

We have

$$
\begin{aligned}
(t- & \left.t_{0}\right)^{1-\alpha}\left|\phi_{n+2}(t)-\phi_{n+1}(t)\right| \\
& \leq\left(t-t_{0}\right)^{1-\alpha} \int_{t_{0}}^{t} \frac{(t-s)^{\alpha-1}}{\Gamma(\alpha)}\left|f\left(s, \phi_{n+1}(s)\right)-f\left(s, \phi_{n}(s)\right)\right| d s \\
& \leq\left(t-t_{0}\right)^{1-\alpha} \int_{t_{0}}^{t} \frac{(t-s)^{\alpha-1}}{\Gamma(\alpha)} L\left(s-t_{0}\right)^{k}\left|\phi_{n+1}(s)-\phi_{n}(s)\right| d s \\
& \leq\left(t-t_{0}\right)^{1-\alpha} \int_{t_{0}}^{t} \frac{(t-s)^{\alpha-1}}{\Gamma(\alpha)} L\left(s-t_{0}\right)^{k} L^{n} M\left(t-t_{0}\right)^{(n+1)+(n+1) k} \prod_{i=1}^{n} \frac{\mathbf{B}(\alpha,(i+1)(k+1))}{\Gamma(\alpha)} d s \\
& =L^{n+1} M\left(t-t_{0}\right)^{(n+2)+(n+2) k} \prod_{i=1}^{n+1} \frac{\mathbf{B}(\alpha,(k+1)(i+1))}{\Gamma(\alpha)} .
\end{aligned}
$$

So the result is correct when $n+1$. Then by the mathematical induction method, we get

$$
\begin{aligned}
\left(t-t_{0}\right)^{1-\alpha}\left|\phi_{n+2}(t)-\phi_{n+1}(t)\right| & \leq L^{n+1} M\left(t-t_{0}\right)^{(n+2)+(n+2) k} \prod_{i=1}^{n+1} \frac{\mathbf{B}(\alpha,(k+1)(i+1))}{\Gamma(\alpha)} \\
& \leq L^{n+1} M h_{2}^{(n+2)+(n+2) k} \prod_{i=1}^{n+1} \frac{\mathbf{B}(\alpha,(k+1)(i+1))}{\Gamma(\alpha)} \\
& =M L^{n+1} h_{2}^{(n+2)(1+k)} \prod_{i=1}^{n+1} \frac{\Gamma((k+1)(i+1))}{\Gamma(\alpha+(i+1)(k+1))}
\end{aligned}
$$

Consider

$$
\sum_{n=1}^{\infty} u_{n}=: \sum_{n=1}^{\infty} M L^{n+1} h_{2}^{(n+2)(1+k)} \prod_{i=1}^{n+1} \frac{\Gamma((k+1)(i+1))}{\Gamma(\alpha+(i+1)(k+1))} .
$$

By a similar method to the one used in the proof of Lemma 2.4, we get

$$
\frac{u_{n+1}}{u_{n}}=L h_{1}^{\alpha+k} \frac{\Gamma((n+2)(k+1))}{\Gamma(\alpha+(n+2)(k+1))} \rightarrow 0 \quad \text { as } n \rightarrow \infty
$$

Then $\sum_{n=1}^{\infty} u_{n}$ is convergent. Hence

$$
\begin{aligned}
& \left(t-t_{0}\right)^{1-\alpha} \phi_{0}(t)+\left(t-t_{0}\right)^{1-\alpha}\left[\phi_{1}(t)-\phi_{0}(t)\right]+\cdots+\left(t-t_{0}\right)^{1-\alpha}\left[\phi_{n}(t)-\phi_{n-1}(t)\right]+\cdots, \\
& \quad t \in\left[t_{0}, t_{0}+h_{2}\right]
\end{aligned}
$$

is uniformly convergent. Then $\left\{\left(t-t_{0}\right)^{1-\alpha} \phi_{n}(t)\right\}$ is convergent uniformly on $\left[t_{0}, t_{0}+h_{2}\right]$. The proof is complete. 
Lemma 2.9 Suppose that (A2) holds and there exists $L>0$ such that $f\left(t,\left(t-t_{0}\right)^{\alpha-1} x_{1}\right)-$ $f\left(t,\left(t-t_{0}\right)^{\alpha-1} x_{2}\right)\left|\leq L\left(t-t_{0}\right)^{k}\right| x_{1}-x_{2} \mid$ for all $t \in\left(t_{0}, t_{0}+h_{1}\right]$ and $x_{1}, x_{2} \in B_{t}$. Then $\phi(t)=(t-$ $\left.t_{0}\right)^{\alpha-1} \lim _{n \rightarrow \infty}\left(t-t_{0}\right)^{1-\alpha} \phi_{n}(t)$ is a unique continuous solution of (6) defined on $\left[t_{0}, t_{0}+h_{2}\right]$.

Proof By $\phi(t)=\left(t-t_{0}\right)^{\alpha-1} \lim _{n \rightarrow \infty}\left(t-t_{0}\right)^{1-\alpha} \phi_{n}(t)$ and Lemma 2.6, we see $\left(t-t_{0}\right)^{1-\alpha} \mid \phi(t)-$ $x_{0} \mid \leq b$. Then

$$
\left|f\left(t, \phi_{n}(t)\right)-f(t, \phi(t))\right| \leq L\left(t-t_{0}\right)^{k}\left(t-t_{0}\right)^{1-\alpha}\left|\phi_{n}(t)-\phi(t)\right|, \quad t \in\left(t_{0}, t_{0}+h_{2}\right] .
$$

It follows that

$$
\begin{aligned}
& \left(t-t_{0}\right)^{-k}\left|f\left(t, \phi_{n}(t)\right)-f(t, \phi(t))\right| \leq L\left(t-t_{0}\right)^{1-\alpha}\left|\phi_{n}(t)-\phi(t)\right| \rightarrow 0 \\
& \text { uniformly as } n \rightarrow \infty \text { on }\left(t_{0}, t_{0}+h_{2}\right] .
\end{aligned}
$$

Hence

$$
\begin{aligned}
\left(t-t_{0}\right)^{1-\alpha} \phi(t) & =\lim _{n \rightarrow \infty} \phi_{n}(t)=\lim _{n \rightarrow \infty}\left[x_{0}+\left(t-t_{0}\right)^{1-\alpha} \int_{t_{0}}^{t} \frac{(t-s)^{\alpha-1}}{\Gamma(\alpha)} f\left(s, \phi_{n-1}(s)\right) d s\right] \\
& =x_{0}+\lim _{n \rightarrow \infty}\left(t-t_{0}\right)^{1-\alpha} \int_{t_{0}}^{t} \frac{(t-s)^{\alpha-1}}{\Gamma(\alpha)} f\left(s, \phi_{n-1}(s)\right) d s \\
& =x_{0}+\left(t-t_{0}\right)^{1-\alpha} \lim _{n \rightarrow \infty} \int_{t_{0}}^{t} \frac{(t-s)^{\alpha-1}}{\Gamma(\alpha)}\left(s-t_{0}\right)^{k}\left(s-t_{0}\right)^{-k} f\left(s, \phi_{n-1}(s)\right) d s \\
& =x_{0}+\left(t-t_{0}\right)^{1-\alpha} \int_{t_{0}}^{t} \frac{(t-s)^{\alpha-1}}{\Gamma(\alpha)}\left(s-t_{0}\right)^{k} \lim _{n \rightarrow \infty}\left(s-t_{0}\right)^{-k} f\left(s, \phi_{n-1}(s)\right) d s \\
& =x_{0}+\left(t-t_{0}\right)^{1-\alpha} \int_{t_{0}}^{t} \frac{(t-s)^{\alpha-1}}{\Gamma(\alpha)} f(s, \phi(s)) d s .
\end{aligned}
$$

It follows that

$$
\phi(t)=x_{0}\left(t-t_{0}\right)^{\alpha-1}+\int_{t_{0}}^{t} \frac{(t-s)^{\alpha-1}}{\Gamma(\alpha)} f(s, \phi(s)) d s
$$

Then $\phi$ is a unique continuous solution of (6) defined on $\left[t_{0}, t_{0}+h_{2}\right]$.

Suppose that $\psi$ defined on $\left(t_{0}, t_{0}+h_{2}\right]$ is also a solution of (6). Then $\left(t-t_{0}\right)^{1-\alpha}|\psi(t)| \leq b$ for all $t \in\left(t_{0}, t_{0}+h_{2}\right]$ and

$$
\psi(t)=x_{0}\left(t-t_{0}\right)^{\alpha-1}+\int_{t_{0}}^{t} \frac{(t-s)^{\alpha-1}}{\Gamma(\alpha)} f(s, \phi(s)) d s, \quad t \in\left(t_{0}, t_{0}+h_{2}\right]
$$

We need to prove that $\phi(t) \equiv \psi(t)$ on $\left(t_{0}, t_{0}+h_{2}\right]$. By (A2), there exist $k>-1$ and $M \geq 0$ such that

$$
|f(t, \psi(t))|=\left|f\left(t,\left(t-t_{0}\right)^{\alpha-1}\left(t-t_{0}\right)^{1-\alpha} \psi(t)\right)\right| \leq M\left(t-t_{0}\right)^{k} \quad \text { for all } t \in\left(t_{0}, t_{0}+h_{2}\right] .
$$


Then

$$
\begin{aligned}
\left(t-t_{0}\right)^{1-\alpha}\left|\phi_{0}(t)-\psi(t)\right| & =\left(t-t_{0}\right)^{1-\alpha}\left|\int_{t_{0}}^{t} \frac{(t-s)^{\alpha-1}}{\Gamma(\alpha)} f(s, \psi(s)) d s\right| \\
& \leq\left(t-t_{0}\right)^{1-\alpha} \int_{t_{0}}^{t} \frac{(t-s)^{\alpha-1}}{\Gamma(\alpha)} M\left(s-t_{0}\right)^{k} d s \\
& =M\left(t-t_{0}\right)^{1+k} \frac{\mathbf{B}(\alpha, k+1)}{\Gamma(\alpha)} .
\end{aligned}
$$

Furthermore, we have

$$
\begin{aligned}
\left(t-t_{0}\right)^{1-\alpha}\left|\phi_{1}(t)-\psi(t)\right| & =\left(t-t_{0}\right)^{1-\alpha}\left|\int_{t_{0}}^{t} \frac{(t-s)^{\alpha-1}}{\Gamma(\alpha)}\left(f\left(s, \phi_{0}(s)\right)-f(s, \psi(s))\right) d s\right| \\
& \leq\left(t-t_{0}\right)^{1-\alpha} \int_{t_{0}}^{t} \frac{(t-s)^{\alpha-1}}{\Gamma(\alpha)} L\left(s-t_{0}\right)^{k}\left|\phi_{0}(s)-\psi(s)\right| d s \\
& \leq\left(t-t_{0}\right)^{1-\alpha} \int_{t_{0}}^{t} \frac{(t-s)^{\alpha-1}}{\Gamma(\alpha)} L\left(s-t_{0}\right)^{k} M\left(s-t_{0}\right)^{1+k} \frac{\mathbf{B}(\alpha, k+1)}{\Gamma(\alpha)} d s \\
& =L M \frac{\mathbf{B}(\alpha, k+1)}{\Gamma(\alpha)} \frac{\mathbf{B}(\alpha, 2 k+2)}{\Gamma(\alpha)}\left(t-t_{0}\right)^{2+2 k} .
\end{aligned}
$$

Now suppose that

$$
\left(t-t_{0}\right)^{1-\alpha}\left|\phi_{n}(t)-\psi(t)\right| \leq L^{n} M\left(t-t_{0}\right)^{(n+1)(k+1)} \prod_{i=1}^{n} \frac{\mathbf{B}(\alpha,(k+1)(i+1))}{\Gamma(\alpha)} .
$$

Then

$$
\begin{aligned}
(t & \left.-t_{0}\right)^{1-\alpha}\left|\phi_{n+1}(t)-\psi(t)\right| \\
& =\left(t-t_{0}\right)^{1-\alpha}\left|\int_{t_{0}}^{t} \frac{(t-s)^{\alpha-1}}{\Gamma(\alpha)}\left(f\left(s, \phi_{n}(s)\right)-f(s, \psi(s))\right) d s\right| \\
& \leq\left(t-t_{0}\right)^{1-\alpha} \int_{t_{0}}^{t} \frac{(t-s)^{\alpha-1}}{\Gamma(\alpha)} L\left(s-t_{0}\right)^{k}\left|\phi_{n}(s)-\psi(s)\right| d s \\
& \leq\left(t-t_{0}\right)^{1-\alpha} \int_{t_{0}}^{t} \frac{(t-s)^{\alpha-1}}{\Gamma(\alpha)} L\left(s-t_{0}\right)^{k} L^{n} M\left(s-t_{0}\right)^{(n+1)(k+1)} \prod_{i=1}^{n} \frac{\mathbf{B}(\alpha,(k+1)(i+1))}{\Gamma(\alpha)} d s \\
& =L^{n+1} M\left(t-t_{0}\right)^{(n+2)(k+1)} \prod_{i=1}^{n+1} \frac{\mathbf{B}(\alpha,(k+1)(i+1))}{\Gamma(\alpha)} .
\end{aligned}
$$

Hence

$$
\begin{aligned}
\left(t-t_{0}\right)^{1-\alpha}\left|\phi_{n+1}(t)-\psi(t)\right| & \leq L^{n} M\left(t-t_{0}\right)^{(n+1)(k+1)} \prod_{i=1}^{n} \frac{\mathbf{B}(\alpha,(k+1)(i+1))}{\Gamma(\alpha)} \\
& \leq L^{n} M h_{1}^{(n+1)(k+1)} \prod_{i=1}^{n} \frac{\mathbf{B}(\alpha,(k+1)(i+1))}{\Gamma(\alpha)} \\
& =L^{n} M h_{1}^{(n+1)(k+1)} \prod_{i=1}^{n} \frac{\Gamma((k+1)(i+1))}{\Gamma(\alpha+(i+1)(k+1))} .
\end{aligned}
$$


From a similar method to the one used in the proof of Lemma 2.3 and the proof of Lemma 2.4, we find that

$$
\sum_{n=1}^{\infty} L^{n} M h_{1}^{(n+1)(k+1)} \prod_{i=1}^{n} \frac{\Gamma((k+1)(i+1))}{\Gamma(\alpha+(k+1)(i+1))}
$$

is convergent. Then

$$
L^{n} M h_{1}^{(n+1)(k+1)} \prod_{i=1}^{n} \frac{\Gamma((k+1)(i+1))}{\Gamma(\alpha+(i+1)(k+1))} \rightarrow 0 \quad \text { as } n \rightarrow \infty .
$$

Then $\lim _{n \rightarrow \infty}\left(t-t_{0}\right)^{1-\alpha} \phi_{n}(t)=\left(t-t_{0}\right)^{1-\alpha} \psi(t)$ uniformly on $\left[t_{0}, t_{0}+h_{2}\right]$. Then $\phi(t) \equiv \psi(t)$ on $\left(t_{0}, t_{0}+h_{2}\right]$. The proof is complete.

Proof of Theorem 2.2 From Lemma 2.6 and Lemma 2.9, $\phi(t)=\left(t-t_{0}\right)^{\alpha-1} \lim _{n \rightarrow \infty}(t-$ $\left.t_{0}\right)^{1-\alpha} \phi_{n}(t)$ is a unique continuous solution of IVP (3) defined on $\left(t_{0}, t_{0}+h_{2}\right]$. The proof is ended.

\section{Examples}

In this section, we present two examples to illustrate the main theorems proved in Section 2.

Example 3.1 Consider the following IVP:

$$
\left\{\begin{array}{l}
{ }^{c} D_{0^{+}}^{3 / 4} x=t^{-1 / 2}(1+x(t)), \quad t>0, \\
x(0)=3 .
\end{array}\right.
$$

Choose $a=10, b=8$. Corresponding to IVP (2), we have $\alpha=3 / 4, x_{0}=3$, and $f(t, x)=$ $t^{-1 / 2}(1+x)$ with $k=-1 / 2$. Using Theorem 2.1, we have $M=\max _{t \in[0,10], x \in[-5,11]} t^{-k} f(t, x)=12$. So

$$
h_{1}=\min \left\{a,\left(\frac{b}{M} \frac{\Gamma(\alpha)}{\mathbf{B}(\alpha, k+1)}\right)^{1 /(\alpha+k)}\right\}=\min \left\{10,\left(\frac{8}{12} \frac{\Gamma(3 / 4)}{\mathbf{B}(3 / 4,1 / 2)}\right)^{4}\right\} .
$$

Then IVP (8) has a unique solution $\phi(t)=\lim _{n \rightarrow \infty} \phi_{n}(t)$ defined on $\left[0, h_{1}\right]$ with

$$
\begin{aligned}
& \phi_{0}(t)=3, \quad t \in\left[0, h_{1}\right] \\
& \phi_{n}(t)=3+\int_{0}^{t} \frac{(t-s)^{-1 / 4}}{\Gamma(3 / 4)} s^{-1 / 2}\left[1+\phi_{n-1}(s)\right] d s, \quad t \in\left[0, h_{1}\right], n=1,2, \ldots
\end{aligned}
$$

Example 3.2 Consider the following IVP:

$$
\left\{\begin{array}{l}
D_{0^{+}}^{1 / 2} x=t^{-2 / 3}\left[1+t(x(t))^{2}\right], \quad t>0 \\
\lim _{t \rightarrow 0} t^{1 / 2} x(t)=3
\end{array}\right.
$$

Choose $a=10, b=8$. Corresponding to IVP (3), we have $\alpha=1 / 2, x_{0}=3$, and $f(t, x)=$ $t^{-2 / 3}\left(1+t x^{2}\right)$ WITH $K=-2 / 3$. Using Theorem 2.2, we have $M=\max _{t \in[0,10], x \in[-5,11]} t^{-k} \times$ 
$f\left(t, t^{\alpha-1} x\right)=122$. So

$$
h_{1}=\min \left\{a,\left(\frac{b}{M} \frac{\Gamma(\alpha)}{\mathbf{B}(\alpha, k+1)}\right)^{1 /(1+k)}\right\}=\min \left\{10,\left(\frac{8}{122} \frac{\Gamma(1 / 2)}{\mathbf{B}(1 / 2,1 / 2)}\right)^{3}\right\} \text {. }
$$

Then IVP (9) has a unique solution $\phi(t)=t^{-1 / 2} \lim _{n \rightarrow \infty} t^{1 / 2} \phi_{n}(t)$ defined on $\left[0, h_{2}\right]$ with

$$
\begin{aligned}
& \phi_{0}(t)=x_{0} t^{-1 / 2}, \quad t \in\left(0, h_{2}\right] \\
& \phi_{n}(t)=3 t^{-1 / 2}+\int_{0}^{t} \frac{(t-s)^{-1 / 2}}{\Gamma(1 / 2)} s^{-2 / 3}\left[1+\left(\phi_{n-1}(s)\right)^{2}\right] d s, \quad t \in\left(0, h_{2}\right], n=1,2, \ldots
\end{aligned}
$$

\section{Conclusions}

In this paper, the initial value problems of fractional differential equations are discussed and new criteria on local existence and uniqueness of solutions are obtained. The wellknown Picard iterative technique is then extended for fractional differential equations which provides computable sequences that converge uniformly to the solution of the problem. We obtain not only the existence and uniqueness of solutions for the problems, but we also establish iterative schemes for approximating the solutions. An example is given to illustrate the main theorems.

Remark 4.1 Theorem 2.1 improves essentially Theorems D and E by generalizing the restrictive condition imposed on $f$. Theorem 2.2 improves the main results in $[4,8,9]$ by removing the assumption that the upper and lower solutions exist. In Theorems 2.1 and 2.2, $t \rightarrow f(t, x)$ or $t \rightarrow f\left(t,\left(t-t_{0}\right)^{\alpha-1} x\right)$ may singular at $t=t_{0}$ (see (A1) and (A2)).

Remark 4.2 In (A1), we may choose $M=\max _{t \in\left[t_{0}, t_{0}+a\right], x \in B}\left(t-t_{0}\right)^{-k} f(t, x)$ if $\left(t-t_{0}\right)^{-k} f(t, x)$ is continuous on $J \times B$. In (A2), one may choose $M=\max _{t \in\left[t_{0}, t_{0}+a\right], x \in B}\left(t-t_{0}\right)^{-k} f\left(t,\left(t-t_{0}\right)^{\alpha-1} x\right)$ if $\left(t-t_{0}\right)^{-k} f\left(t,\left(t-t_{0}\right)^{\alpha-1} x\right)$ is continuous on $J \times B$.

\section{Competing interests}

The authors declare that they have no competing interests.

Authors' contributions

Each of the authors, $X Y$ and $Y L$ contributed to each part of this study equally and read and approved the final version of the manuscript.

\section{Author details}

${ }^{1}$ Department of Computers, Guangdong Police College, Guangzhou, 510230, P.R. China. ²Department of Mathematics, Guangdong University of Finance and Economics, Guangzhou, 510320, P.R. China.

\section{Acknowledgements}

The article is supported by the Natural Science Foundation of Guangdong province (No: S2011010001900) and the Guangdong Higher Education Foundation for High-level talents. The authors would like to thank the referees for their careful reading and some useful comments on improving the presentation of this paper.

Received: 9 December 2013 Accepted: 18 March 2014 Published: 3 April 2014

\section{References}

1. Miller, KS, Ross, B: An Introduction to the Fractional Calculus and Fractional Differential Equation. Wiley, New York (1993)

2. Podlubny, I: Fractional Differential Equations. Academic Press, New York (1999)

3. Lakshmikantham, V: Theory of fractional functional differential equations. Nonlinear Anal. 69, 3337-3343 (2008)

4. Lakshmikantham, V, Vatsala, AS: General uniqueness and monotone iterative technique for fractional differential equations. Appl. Math. Lett. 21, 828-834 (2008)

5. Lakshmikantham, V, Vatsala, AS: Basic theory of fractional differential equations. Nonlinear Anal. 69, 2677-2682 (2008) 
6. Lin, W: Global existence theory and chaos control of fractional differential equations. J. Math. Anal. Appl. 332, 709-726 (2007)

7. Zhou, Y: Existence and uniqueness of solutions for a system of fractional differential equations. Fract. Calc. Appl. Anal. 12(2), 195-204 (2009)

8. Zhang, S: Monotone iterative method for initial value problem involving Riemann-Liouville fractional derivatives. Nonlinear Anal. 71, 2087-2093 (2009)

9. Tazali, A-AM: Local existence theorems for ordinary differential equations of fractional order. In: Ordinary and Partial Differential Equations, Dundee, 1982. Lecture Notes in Math., vol. 964, pp. 652-665 (1982)

10. Kilbas, AA, Trujillo, JJ: Differential equations of fractional order: methods results and problem, I. Appl. Anal. 1(78), 153-192(2001)

doi:10.1186/1687-1847-2014-102

Cite this article as: Yang and Liu: Picard iterative processes for initial value problems of singular fractional differential equations. Advances in Difference Equations 2014 2014:102

Submit your manuscript to a SpringerOpen ${ }^{\circ}$ journal and benefit from:

- Convenient online submission

- Rigorous peer review

- Immediate publication on acceptance

- Open access: articles freely available online

- High visibility within the field

- Retaining the copyright to your article

Submit your next manuscript at $>$ springeropen.com 